\title{
Challenges in Cartilage Tissue Engineering
}

\section{Portocarrero G, Collins $\mathbf{G}$ and Livingston Arinzeh $\mathbf{T}^{*}$}

Department of Biomedical Engineering, New Jersey Institute of Technology, Newark, NJ 07102, USA

\section{The Problem}

Over 27 million Americans suffer from cartilage damage with several million people affected per year. Articular cartilage or hyaline cartilage has a limited capacity to heal after damage from injury or degenerative disease. In an attempt to delay the progression of widespread damage, orthopedic surgeons routinely treat osteochondral injuries with minimally invasive surgical procedures involving articular resurfacing. The knee is the most prevalent joint affected. One of the three most common treatments for injury of the knee is arthroscopic lavage, either with or without debridement. Yet this treatment is merely palliative, providing symptomatic relief but does not repair injury to cartilage [1]. The most frequently used reparative treatment for small symptomatic lesions of articular cartilage of the knee are marrow-stimulating techniques, such as subchondral drilling, abrasion arthroplasty and microfracturin [2]. In microfracturing, which is the most commonly used technique, multiple holes made in the subchondral bone allow bone marrow components to reach the joint surface and facilitate repair. Bone marrow contains mesenchymal stem cells that have the potential to form new cartilage. However, fibrocartilage typically forms, which is mechanically much less robust than articular cartilage. While it may provide pain mitigation, fibrocartilage typically shows signs of degeneration after one year [3,4]. Alternative surgical procedures include autologous chondrocyte transplantation and osteoarticular transfer. These procedures require harvesting cartilage from the patient or cadaveric source and later injecting/grafting into the lesion. In the past few years, three systematic reviews of current treatments for focal chondral defects have come to the same conclusion that no "gold standard" exists for repairing chondral injuries [2,5,6]. All current surgical interventions produce mediocre efficacy. Once degeneration has progressed to severe osteoarthritis (OA) where articular resurfacing is no longer an option, the only recourse is Total Knee Replacement (TKR). However, TKR is contraindicated for patients younger than 45 -years old, for whom no alternative treatment exists. This younger patient population is increasing due to sports-related injuries and the growing obesity epidemic. Therefore, a growing unmet need exists for future interventions that dramatically delay or halt widespread cartilage damage.

\section{Issues That Make Cartilage Repair a Difficult Problem}

A large number of reported efforts in tissue engineering to regenerate cartilage tissue have demonstrated the success of these methods in growing chondrocytes or undifferentiated cells alone or in combination with various types of three-dimensional scaffolds and hydrogels fabricated from natural or synthetic materials. While in some cases initial formation of cartilage-like tissue is reported, no approach to date has produced a regenerated tissue with long-term stability. Currently, the quality and function of many tissue-engineered therapies still needs to be improved to fully address the clinical need. Autologous chondrocyte transplantation, which is clinically available, involves the use of autologous cultured chondrocytes. A patient's chondrocytes are expanded, injected into a cartilage defect site, and held in place with a periosteal patch. Of major concern is the limited proliferative capacity of differentiated chondrocytes in providing adequate cell numbers for transplantation and their proliferative capacity decreases with patient age. Long-term studies in patients also have demonstrated that these treated defects are filled with fibrocartilage as opposed to normal hyaline cartilage. A product currently undergoing phase III clinical trials, Neocartilage Implant/DeNovo ET (Engineered Tissue Graft), an implant developed from cultured human juvenile chondrocytes, is showing promise. Juvenile chondrocytes, obtained from 13-yearold or younger human cadaveric donors, was shown to produce more extracellular matrix than adult chondrocytes, which may be considered an important characteristic for efficient cartilage repair $[7,8]$. The greater regenerative capacity of these cells, producing robust extracellular matrix (ECM), may improve overall mechanical stability of the tissue and provide ECM components necessary for the maintenance of the chondrocyte phenotype. However, juvenile chondrocytes as a cell source may have limitations due to donor availability.

It is likely that a successful tissue regeneration approach would have to encourage cells to produce the cartilage ECM or provide a materials based scaffold that mimics these elements. The ECM of hyaline cartilage can be considered a fiber reinforced hydrogel composite material. The reinforcing fiber is the fibrous collagen protein distributed in the proteoglycan hydrogel matrix. The ability of cartilage to withstand both the tensile and compressive loading it experiences is related to its composite material properties. Two critical features of fiber-reinforced composites exist that relate directly to mechanical behavior. One is the orientation of the fiber with respect to the load, and the other is the transmission of the load from the low modulus matrix to the high modulus fiber reinforcement. In native articular cartilage, there is a complex spatial distribution of fiber orientation that varies from the articular surface to the subchondral bone interface. This distribution facilitates the management of those tensile and compressive loads. In order to transmit the load from the matrix to the fiber, a strong interfacial bond must be accomplished between the hydrogel matrix and protein fiber phases. A subtle feature of Type II collagen in articular cartilage is the "decoration" of its surface with Type IX collagen [9]. Type IX collagen chemically binds chondroitin sulfate providing a facile interaction between the fibrous protein reinforcement and the proteoglycan matrix. The fiber spatial orientation distribution and the fiber-matrix interface are fundamental elements of composite material behavior. In addition, the chondroitin sulfate and other sulfated glycosaminoglycans contained within the matrix interact with growth factors and help maintain their bioactivity,

*Corresponding author: Treena Livingston Arinzeh, PhD, Department of Biomedical Engineering, New Jersey Institute of Technology, University Heights, 614 Fenster Hall, Newark, NJ 07102-1982, USA, Tel: +1-973-5965269; Fax: +1-973-596-5222; E-mail: arinzeh@njit.edu

Received December 22, 2012; Accepted December 24, 2012; Published December 26, 2012

Citation: Portocarrero G, Collins G, Livingston Arinzeh T (2013) Challenges in Cartilage Tissue Engineering. J Tissue Sci Eng 4:e120. doi:10.4172/21577552.1000 e120

Copyright: (c) 2013 Portocarrero G, et al. This is an open-access article distributed under the terms of the Creative Commons Attribution License, which permits unrestricted use, distribution, and reproduction in any medium, provided the original author and source are credited. 
which is important for promoting/maintaining cell function. The subtle features of articular cartilage give it the unique mechanical and biological behavior observed. An equally challenging issue will be the integration of the engineered tissue into the native host tissue. Considering the mechanical environment, engineered tissue securely anchored by long-term physiological integration rather than surgical attachment would be needed.

\section{Future of Cartilage Tissue Engineering}

Stem cells, if they are provided the appropriate cues to differentiate and can produce the required articular cartilage ECM, may be a promising cell source in cartilage tissue engineering due to their availability. Adult derived stem cells, which are obtained from adult tissue or from adult somatic cells which can be transformed to induced pluripotent stem cells, appear promising. Combining stem cells with scaffolds that provide the appropriate inductive cues as well as structural properties may be necessary for their success. Scaffolds are also being investigated in combination with the surgical method of micro fracturing to coax the patient's own bone marrow stem cells to form articular cartilage. However, cartilage is distinct as a tissue in that its function is not apparently related to an on-going, high level of cellular activity. Articular cartilage functions as a critical mechanical interface, and the cells native to the tissue function minimally to maintain mechanical integrity. It is not unreasonable to consider that future approaches to cartilage repair may not depend on cellular regeneration strategies. Instead, in a manner similar the development of intraocular implants, the native tissue could be replaced with a fiber reinforced hydrogel engineered to provide cartilage-like biological performance.

\section{References}

1. Rönn K, Reischl N, Gautier E, Jacobi M (2011) Current surgical treatment of knee osteoarthritis. Arthritis 2011: 454873.

2. Bedi A, Feeley BT, Williams RJ 3rd (2010) Management of articular cartilage defects of the knee. J Bone Joint Surg Am 92: 994-1009.

3. Hunziker EB (2002) Articular cartilage repair: basic science and clinical progress. A review of the current status and prospects. Osteoarthritis Cartilage 10: $432-463$

4. Temenoff JS, Mikos AG (2000) Review: tissue engineering for regeneration of articular cartilage. Biomaterials 21: 431-440.

5. Benthien JP, Schwaninger M, Behrens $P$ (2011) We do not have evidence based methods for the treatment of cartilage defects in the knee. Knee Surg Sports Traumatol Arthrosc 19: 543-552.

6. Khashan M, Chechik O, Arbel R, Morag G (2010) The treatment of focal chondral lesions of the knee. Harefuah 149: 542-546, 549.

7. Adkisson HD 4th, Martin JA, Amendola RL, Milliman C, Mauch KA, et al. (2010) The potential of human allogeneic juvenile chondrocytes for restoration of articular cartilage. Am J Sports Med 38: 1324-1333.

8. Alsat E, Wyplosz P, Malassiné A, Guibourdenche J, Porquet D, et al. (1996) Hypoxia impairs cell fusion and differentiation process in human cytotrophoblast, in vitro. J Cell Physiol 168: 346-353.

9. Eyre DR, Pietka T, Weis MA, Wu JJ (2004) Covalent cross-linking of the NC1 domain of collagen type IX to collagen type II in cartilage. J Biol Chem 279 2568-2574. 